\title{
On the formation and properties of fluid shocks and collisionless shock waves in astrophysical plasmas
}

\author{
Antoine Bret ${ }^{1,2, \dagger}$ and Asaf Pe'er ${ }^{3}$ \\ ${ }^{1}$ ETSI Industriales, Universidad de Castilla-La Mancha, 13071 Ciudad Real, Spain \\ ${ }^{2}$ Instituto de Investigaciones Energéticas y Aplicaciones Industriales, \\ Campus Universitario de Ciudad Real, 13071 Ciudad Real, Spain \\ ${ }^{3}$ Physics Department, University College Cork, Cork, Ireland
}

(Received 26 March 2018; revised 25 May 2018; accepted 29 May 2018)

When two plasmas collide, their interaction can be mediated by collisionless plasma instabilities or binary collisions between particles of each shell. By comparing the maximum growth rate of the collisionless instabilities with the collision frequency between particles of the shells, we determine the critical density separating the collisionless formation from the collisional formation of the resulting shock waves. This critical density is also the density beyond which the shock downstream is field free, as plasma instabilities do not have time to develop electromagnetic patterns. We further determine the conditions on the shells initial density and velocity for the downstream to be collisional. If these quantities fulfil the determined conditions, the collisionality of the downstream also prevents the shock from accelerating particles or generating strong magnetic fields. We compare the speed of sound with the relative speed of collision between the two shells, thus determining the portion of the parameter space where strong shock formation is possible for both classical and degenerate plasmas. Finally, we discuss the observational consequences in several astrophysical settings.

Key words: astrophysical plasmas, plasma instabilities, space plasma physics

\section{Introduction}

Shock waves are among the most ubiquitous and most studied physical phenomena. They exist in many different astronomical objects, at very many different scales. They play a major role in shaping the observed signal of various objects, providing (i) a natural way of depositing kinetic energy; (ii) the necessary conditions for acceleration of particles to high energies, non-thermal distribution; and furthermore, (iii) shock waves may be responsible for generating strong magnetic fields (Bell 1978; Silva et al. 2003; Spitkovsky 2008; Sironi \& Spitkovsky 2011). 
Shock waves may come in two flavours. In a neutral fluid, kinetic energy dissipation at the shock front is provided by binary collisions. ${ }^{1}$ As a result, the shock front is a few mean-free-paths thick (Zel'dovich \& Raizer 2002), and the shock is 'collisional'. In charged plasma, on the other hand, instabilities prompted by collective behaviour can equally mediate shock waves and provide the kinetic energy dissipation at the front (Petschek 1958; Sagdeev 1966). In this case, the shock front can be several orders of magnitudes shorter than the mean-free-path for binary collisions (Bamba et al. 2003). These shocks have been dubbed 'collisionless shocks'.

While there were still doubts about the very existence of collisionless shocks in the late 1980s (Sagdeev \& Kennel 1991), in situ observations of the Earth bow shock, for example, have definitely cast them out (Bale, Mozer \& Horbury 2003; Schwartz et al. 2011). Because they are collisionless, these shocks are formed through collective plasma instabilities, on the time scale of these instabilities (Bret et al. 2013, 2014).

The absence of close collisions allows particles to gain energy without sharing it with others. As a result, collisionless shocks are excellent particle accelerators (Blandford \& Eichler 1987; Marcowith et al. 2016), as opposed to collisional shocks (e.g. Longair 2011).

In view of the properties which derive from the absence of collisions, one can wonder about the conditions required for collisionless-ness to be fulfilled. The goal of this paper is to investigate (i) the conditions required for the shock formation to be collisionless, and (ii) the conditions required for the downstream region to be so.

Regarding the first item, the nature of the shock formation determines the time scale on which it forms. In the collisionless regime, the two colliding shells start passing through each other. The overlapping region quickly turns unstable, generating a turbulence which blocks the flow and triggers the shock formation (Bret et al. 2013, 2014). Binary collisions between the particles of each shell can be neglected if the (average) collision frequency $v_{s s}$ is much smaller than the growth rate $\delta$ of the fastest counter-streaming instability involved in the overlapping region. On the other hand, if $\delta \ll v_{s s}$, binary collisions govern the dynamics that the shells encounter. We thus find that a quantitative investigation of the interface between the collisional and the collisionless regimes comes down to comparing $\delta$ and $v_{s s}$. Note that such an endeavour only makes sense in a plasma, for in a non-ionized collisionless medium, counterstreaming flows are stable $(\delta=0)$ and can only be disrupted by binary collisions.

Still, for the first item, the nature of the shock formation determines the electromagnetic patterns that will be found in the downstream, once the shock is formed. Such patterns, like Weibel filaments (Milosavljevic \& Nakar 2006; Huntington et al. 2015), are the fruit of plasma instabilities in the collisionless case. If the shock is formed through close binary collisions, these instabilities will not grow and will not be able to seed electromagnetic patterns in the downstream. In turn, the absence of fields in the downstream means the absence of scattering agents for the particles, inhibiting their acceleration.

As for the ability of the shock to accelerate particles, we point out that the time scale required for Fermi acceleration is much longer than that required for shock formation. Therefore, even a weak collisionality could allow for collisionless shock formation, while suppressing acceleration. As a result, the limit for acceleration set below, namely by 'inter-shell collision frequency = growth rate', could indeed be an upper bound, as acceleration could be cancelled even slightly before this threshold.

\footnotetext{
${ }^{1}$ We focus the discussion here on shocks that propagate in an environment in which the energy density of radiation downstream can be neglected with respect to the particle thermal energy.
} 
Regarding the second shock item investigated if this paper, namely, the collisionality of the downstream, it also determines whether or not the shock, once formed, is capable of accelerating particles. The reason for this comes from the fact that particle acceleration in a Fermi process results from back-and-forth motions around the shock front (Spitkovsky 2008). This is only possible if both the upstream and the downstream are collisionless, so that particles can nearly freely travel between each region, without exchanging energy with the others. But if the downstream happens to be collisional, particles will be trapped inside as soon as they enter it. They will remain embedded into the downstream flow, constantly exchanging energy with the others, and unable to keep it or to close any Fermi acceleration cycle.

While the shock compresses the gas, it also compresses any parallel magnetic field. Thus, while the density in the downstream region is higher than in the upstream region, particles accelerated by a Fermi mechanism can spend more time in the upstream region. Thus, in determining the ability of the shock to support acceleration of particles to high energies, one needs to probe the conditions in both the upstream and downstream regions.

Nonetheless, as the compression ratio of the density is similar to that of the parallel component of the magnetic field, the ratio of Larmor radii in the upstream and downstream regions cannot exceed the ratio of downstream to upstream densities. As a consequence, the analysis of the conditions at the upstream region is not expected to affect the results obtained by analysing the downstream region alone by a factor larger than two.

This paper is structured as follow. We begin by considering pair plasmas in $\S 2$. In $\S 2.1$ we calculate the collision frequency $v_{s s}$ for close Coulomb collisions between particles of the two pair shells. We then compute in $\S 2.2$ the growth rate $\delta$ of the fastest growing collisionless mode. In $\S 2.3, v_{s s}$ and $\delta$ are compared, allowing us to determine the portions of the phase space $\left(\gamma_{0}, n_{0}\right)$ (initial Lorentz factor and density of the colliding plasma shells) where the shock formation is mediated by collisionless plasmas effects, or inter-shell binary collisions. Finally, $\S 2.4$ derives the requirements on $\left(\gamma_{0}, n_{0}\right)$ for the downstream of the shock, once formed, to be collisional.

We then turn to electron/proton plasmas in $\S 3$. We conduct similar calculations as for the pair plasmas case, emphasizing the qualitative difference that results from the difference in instability growth rate in this scenario. Following these theoretical derivations, we explore the limits of the small velocity spread approximation within each shell used throughout this work in $\S 4$. By equating the speed of sound in the different regimes (classical and quantal gas, Newtonian and highly relativistic) to the speed of collision between the shells, we constrain the parameter space region in which strong shocks can possibly form. Finally, we discuss in $\S 5$ the conditions that exist inside several astronomical objects, and the applicability of the theory to these various objects, before we reach our conclusions in $\S 6$.

\section{Colliding pair plasma shells}

We begin by considering the scenario of two symmetric plasma shells composed of electron-position pairs heading toward each other, as pictured in figure 1. Each shell is initially cold (see discussion in $\S 4$ ), with initial (laboratory frame) density $n_{0}$ and Lorentz factor $\gamma_{0}=\left(1-\beta_{0}^{2}\right)^{-1 / 2}$, where $\beta_{0}=v_{0} / c$ is the normalized flow velocity. 

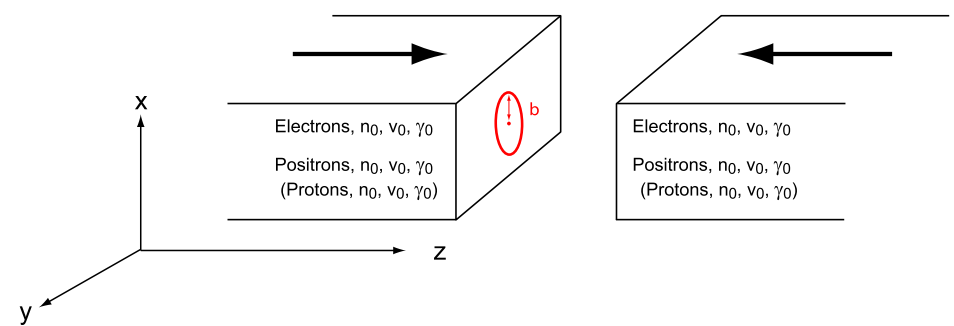

FIGURE 1. System considered: two counter-streaming plasmas collide. They are initially cold and symmetric, with electronic density $n_{0}$ in the laboratory frame. The first part of the article deals with pair plasmas, while the second part deals with electron/proton plasmas.

\subsection{Inter-shell collision frequency}

The impact parameter for close binary Coulomb collisions is defined via (see equation (13.4) in Jackson (1998), with deviation $\theta=\pi / 2$ )

$$
b_{C}=\frac{q^{2}}{\gamma_{r} m_{e} v_{r}^{2}},
$$

where $\gamma_{r}$ is the relative Lorentz factor of the two shells, namely $\gamma_{r}=2 \gamma_{0}^{2}-1$. The relative velocity of the two shells is

$$
v_{r}=\frac{2 \beta_{0}}{1+\beta_{0}^{2}} c,
$$

where clearly $\gamma_{r}=\left(1-v_{r}^{2} / c^{2}\right)^{-1 / 2}$. When $b_{C}$ becomes too small, it has to be replaced by the relevant de Broglie length (see equation (5.10) in Rybicki \& Lightman (1979)),

$$
b_{Q}=\frac{\hbar}{p}=\frac{\hbar}{\gamma_{r} m_{e} v_{r}} .
$$

The frequency for close collisions between particles of two different shells then reads,

$$
v_{s s}=n_{0} v_{r} \pi b^{2}=n_{0} \frac{2 \beta_{0}}{1+\beta_{0}^{2}} c \pi \max \left(b_{C}, b_{Q}\right)^{2} .
$$

Since $b_{C}$ is proportional to $v_{r}^{-2}$ and $b_{Q} \propto v_{r}^{-1}$, the impact parameter is classical at low velocity, and quantum at high velocity. The two values of the impact parameters become equal at

$$
\frac{q^{2}}{\gamma_{r} m_{e} v_{r, e q .}^{2}}=\frac{\hbar}{\gamma_{r} m_{e} v_{r, e q .}} \Rightarrow \frac{v_{r, e q .}}{c} \equiv \beta_{r, e q .}=\frac{q^{2}}{\hbar c}=\alpha,
$$

namely at sub-relativistic velocities. Here $\alpha \sim 1 / 137$ is the fine structure constant.

In terms of $\beta_{0}$ and $\gamma_{0}$, equality is achieved for

$$
\left.\begin{array}{c}
\gamma_{0, e q .} \equiv \gamma_{0}^{*}=\sqrt{\frac{\gamma_{r, e q .}+1}{2}} \sim 1.0000067, \\
\beta_{0, e q .} \equiv \beta_{0}^{*}=\sqrt{1-1 / \gamma_{0}^{* 2}} \sim 0.00365 .
\end{array}\right\}
$$

We thus find that for $\beta_{0}<\beta_{0}^{*}$, the frequency of close inter-shell collisions is given by (2.4) with $\max \left(b_{C}, b_{Q}\right)=b_{C}$. For $\beta_{0}>\beta_{0}^{*}$, it is given by (2.4) with $\max \left(b_{C}, b_{Q}\right)=b_{Q}$. 


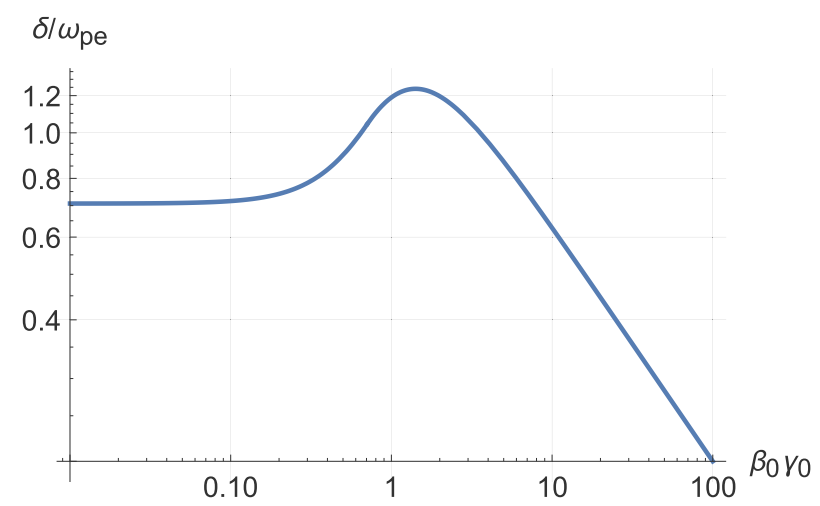

FIGURE 2. Growth rate of the fastest growing mode in terms of $\beta_{0} \gamma_{0}$ for cold pair plasmas interaction.

\subsection{Maximum instability growth rate $\delta$}

In collisionless plasmas, shock formation is triggered by the counter-streaming instabilities that arise when the shells start overlapping. These instabilities are numerous and can be found for wave vectors aligned, normal or even oblique to the flow (Watson, Bludman \& Rosenbluth 1960; Bret, Gremillet \& Dieckmann $2010 b$ ). For the present case, the growth rate of the fastest growing mode is only a function of the Lorentz factor. It has already been determined for any $\gamma_{0}$ in Bret et al. (2010b); for completeness, we here recall the main results. It is noteworthy that the forthcoming growth rates are analytically exact.

For $\gamma_{0}>\sqrt{3 / 2}\left(\beta_{0}>1 / \sqrt{3}\right)$, the fastest growing mode is the Weibel mode (Bret et al. 2013), with $\boldsymbol{k} \perp \boldsymbol{v}_{0}$. Its growth rate is

$$
\delta=2 \frac{\beta_{0}}{\sqrt{\gamma_{0}}} \omega_{p e},
$$

where $\omega_{p e}=\sqrt{4 \pi n_{0} q^{2} / m_{e}}$ is the (electron) plasma frequency. In the opposite regime $\gamma_{0}<\sqrt{3 / 2}$, the fastest growing mode is oblique, with growth rate

$$
\delta=\left(1+\beta_{0}^{2}\right) \sqrt{\frac{\gamma_{0}}{2}} \omega_{p e} .
$$

Expressions (2.7) and (2.8) are plotted together as functions of $\beta_{0} \gamma_{0}$ in figure 2 . The threshold $\gamma_{0}=\sqrt{3 / 2}$ corresponds to $\beta_{0} \gamma_{0}=1 / \sqrt{2}$. The growth rate $\delta$ obtains a maximum value $\delta / \omega_{p e}=2^{3 / 2} / 3^{3 / 4}=1.24$ for $\gamma_{0}=\sqrt{3}\left(\beta_{0} \gamma_{0}=\sqrt{2}\right)$.

The existence of a maximum value for the growth rate $\delta$ can intuitively be understood as follows. For plasmas having Lorentz factor $\gamma_{0}=\sqrt{3}$ the dominant instability is the Weibel instability ( $\operatorname{since} \sqrt{3}>\sqrt{3 / 2}$ ). This instability is driven by the repulsion of opposite currents (Medvedev et al. 2005). It relies therefore on the Lorentz force being $\propto v_{0}$. As a result, it weakens at low velocities. Furthermore, this instability weakens at high velocities as well, since $v_{0}$ cannot surpass $c$ while the relativistic inertia keeps increasing with $\gamma_{0}$. These two features are reflected in the scaling of the growth rate (2.7), which varies like $\beta_{0} / \sqrt{\gamma_{0}}$. With a null limit both at high and low velocities, an intermediate extremum is necessary. Solving $\partial \delta / \partial \gamma_{0}=0$ gives $\gamma_{0}=\sqrt{3}$. 


\subsection{Comparison of collision rate $v_{s s}$ and instability growth rate $\delta$}

In order to determine whether the shock formed is collisional or collisionless, one needs to compare the frequency for close Coulomb collisions with the fastest growth rate given above. One singles out three intervals:

(i) For $\beta_{0}<\beta_{0}^{*}$, the impact parameter is classical. The relevant growth rate in this regime is given by (2.8). In this scenario, one compares (2.4) with $b_{C}>b_{Q}$ and (2.8). The calculation gives

$$
\frac{v_{s s}}{\delta}=\sqrt{\frac{\pi}{128}} \sqrt{\frac{n_{0}}{N^{*}}} \frac{1}{\beta_{0}^{3} \gamma_{0}^{9 / 2}} \simeq \sqrt{\frac{\pi}{128}} \sqrt{\frac{n_{0}}{N^{*}}} \frac{1}{\beta_{0}^{3}} .
$$

Here,

$$
N^{*} \equiv\left(\frac{m c^{2}}{q^{2}}\right)^{3}=4.5 \times 10^{37} \mathrm{~cm}^{-3} .
$$

(ii) For $\beta_{0}>\beta_{0}^{*}$ and $\gamma_{0}<\sqrt{3 / 2}$, the impact parameter is quantum, while the relevant growth rate is still given by (2.8). Comparing (2.4) but now with $b_{C}<b_{Q}$ and (2.8) gives

$$
\frac{v_{s s}}{\delta}=\sqrt{\frac{\pi}{8}} \sqrt{\frac{n_{0}}{N_{1}^{*}}} \frac{1}{\gamma_{0}^{9 / 2} \beta_{0}\left(1+\beta_{0}^{2}\right)^{2}} \simeq \sqrt{\frac{\pi}{8}} \sqrt{\frac{n_{0}}{N_{1}^{*}}} \frac{1}{\beta_{0}} .
$$

Here,

$$
N_{1}^{*}=\frac{m c^{2}}{q^{2}} a_{0}^{-2}=1.26 \times 10^{29} \mathrm{~cm}^{-3},
$$

and $a_{0}=\hbar^{2} / m q^{2}$ is the Bohr radius.

(iii) For $\gamma_{0}>\sqrt{3 / 2}$, the impact parameter is quantum, while the relevant growth rate in this case is given by (2.7). Comparing (2.4) with $b_{C}<b_{Q}$ and (2.7) gives

$$
\frac{v_{s s}}{\delta}=\frac{\sqrt{\pi}}{8} \sqrt{\frac{n_{0}}{N_{1}^{*}}} \frac{1}{\gamma_{0}^{7 / 2} \beta_{0}^{2}\left(1+\beta_{0}^{2}\right)} \simeq \frac{\sqrt{\pi}}{16} \sqrt{\frac{n_{0}}{N_{1}^{*}}} \frac{1}{\gamma_{0}^{7 / 2}} .
$$

One thus concludes that in all 3 cases, the ratio $v_{s s} / \delta$ has the form

$$
\frac{v_{s s}}{\delta}=F \times \sqrt{\frac{n_{0}}{N_{i}^{*}}},
$$

where $N_{i}^{*}=N^{*}$ or $N_{1}^{*}$, depending on the scenario considered. The value of the function $F=F\left(\beta_{0}\right)$ is defined through equations (2.9), (2.11), (2.13) and depends on the scenario considered. We thus conclude that the shock is collisional if

$$
\frac{v_{s s}}{\delta}>1 \Rightarrow n_{0}>\frac{N_{i}^{*}}{F^{2}} .
$$

Figure 3 pictures the critical density beyond which the interaction is collisional. The lower part of the $\left(\gamma_{0}, n_{0}\right)$ phase space pertains to collisionless interactions. 


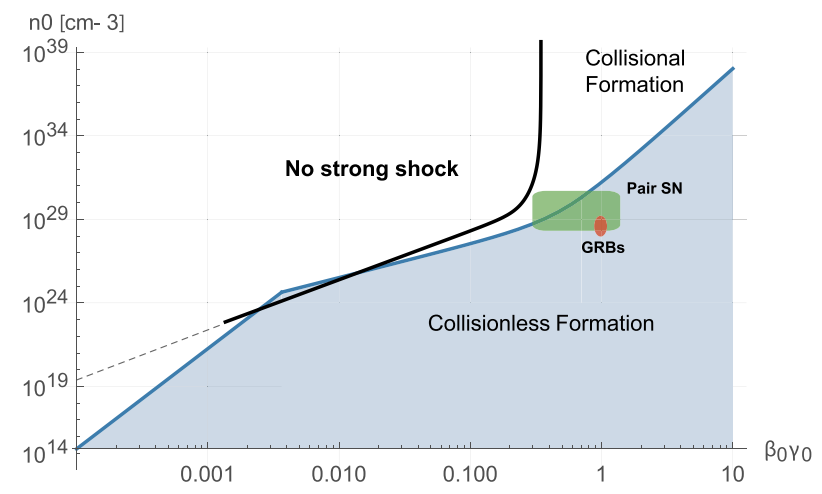

FIGURE 3. Critical density beyond which the interaction is collisional for pair plasmas. The blue shaded area of the phase space pertains to collisionless interactions. The coloured regions pertain to astrophysical scenarios discussed in $\S 5$. The bold black line pictures the no strong shock condition discussed in $\S 4$. As discussed in that section, for $T<T_{F}$ (the Fermi temperature) strong shocks cannot be formed above this line, because the speed of sound is larger than the collision speed.

\subsection{Conditions on $n_{0}$ and $\gamma_{0}$ for a collisionless downstream}

After the two shells collide, a shock is formed with a downstream having density $n$ and temperature $T$. Depending on $n$ and $T$, the downstream region can be collisionless or collisional - even if initially the shock is formed collisionlessly.

A collisionless plasma is weakly coupled. Indeed, by definition, in a weakly coupled plasma the kinetic energy of the plasma's particles is much larger than the potential energy associated with Coulomb collisions. This, in turn, implies that close Coulomb collisions are rare. The parallel extends even to the degenerate regime, as, for example, the Fermi energy of a weakly coupled degenerate plasma can be found assuming free wave functions (plane waves $\propto \mathrm{e}^{\mathrm{i} k \cdot r}$ ) for the particles (Ashcroft \& Mermin 1976).

Figure 4 shows the different plasma regimes in the downstream region, in terms of its temperature and density $(T, n)$. The line $T=T_{F}$ pictures plasmas with temperature equal to the Fermi temperature. Below this line, $T>T_{F}$ and the plasma is classical. It is therefore weakly coupled if its kinetic energy is greater than the Coulomb potential, i.e. $k_{B} T>q^{2} n^{1 / 3}$. $^{2}$ Above this line, $T<T_{F}$ and the plasma is degenerate. It is therefore weakly coupled if the Fermi energy is greater than the Coulomb potential, $k_{B} T_{F}>$ $q^{2} n^{1 / 3}$ (Ichimaru 1982). As the Fermi temperature $T_{F}$ only depends on the density, this latter condition defines a critical density threshold,

$$
\left.\begin{array}{c}
k_{B} T_{F}=\left(3 \pi^{2} n\right)^{2 / 3} \frac{\hbar^{2}}{2 m_{e}}=q^{2} n^{1 / 3} \\
>\frac{8}{9 \pi^{4}}\left(\frac{m_{e} q^{2}}{\hbar^{2}}\right)^{3}=6.3 \times 10^{22} \mathrm{~cm}^{-3},
\end{array}\right\}
$$

instead of an oblique line. As a result, plasmas located inside the red triangle pictured in figure 4 are strongly coupled, i.e. collisional. If the downstream lies in this domain, the shock cannot accelerate particles. Note that at higher densities, the plasma remains

\footnotetext{
${ }^{2}$ Note that in calculating the Coulomb potential there is a prefactor of order unity, which is omitted for clarification.
} 


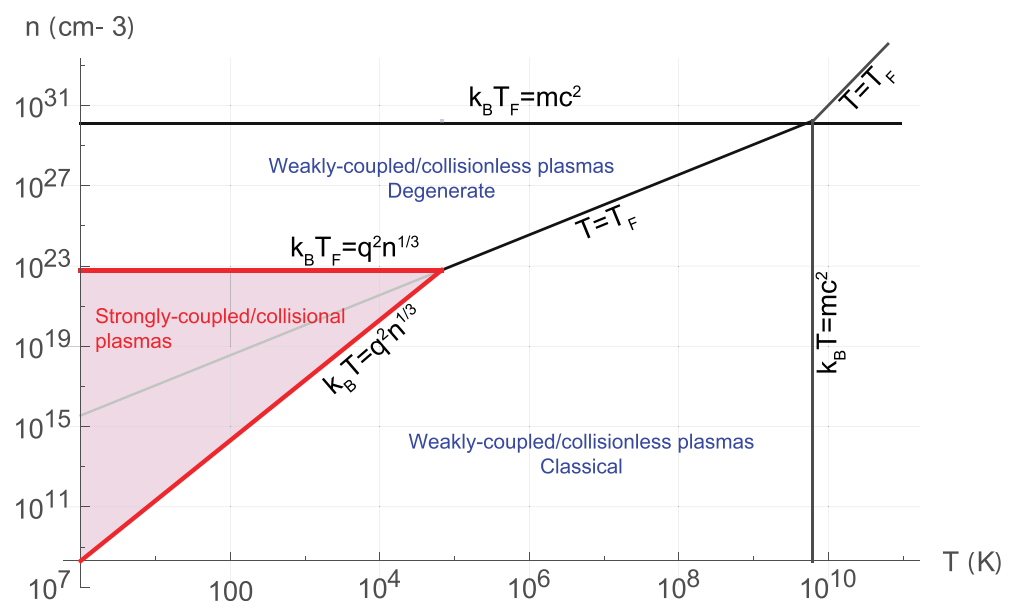

FIgURE 4. Collisionless region in the $(T, n)$ phase space. The red shaded area pertains to strongly coupled plasmas, i.e. collisional plasmas. A classical plasma becomes relativistic for $k_{B} T>m c^{2}$, and a degenerate plasma becomes relativistic for $k_{B} T_{F}>m c^{2}$.

collisionless, somewhat counter-intuitively, as it is kept degenerate as the densities increase, until reaching the relativistic limit. Thus, the rest of the phase parameter space is weakly coupled, whether for classical or quantum reasons.

One can notice that the collisional regime has an upper bound, both in temperature and density. For $T>10^{5} \mathrm{~K}$, or $n>6.3 \times 10^{22} \mathrm{~cm}^{-3}$, the plasma cannot be collisional. Rather, it must be collisionless.

Given that both $n$ and $T$ are functions of the initial density and Lorentz factors $n_{0}$ and $\gamma_{0}$, one can determine the requirements on the initial plasma parameters $\left(n_{0}, \gamma_{0}\right)$ that result in collisional downstream region. From the discussion above, it follows that there are two requirements: (i) for classical plasma regime, it is $k_{B} T \geqslant q^{2} n^{1 / 3}$; (ii) for the quantum border of the collisional regime, one requires that the plasma density is $n>6.3 \times 10^{22} \mathrm{~cm}^{-3}$. We therefore explore the conditions on $n\left(n_{0}, \gamma_{0}\right)$ and $T\left(n_{0}, \gamma_{0}\right)$ that fulfil these requirements.

\subsubsection{Conditions for the downstream to be collisional}

We derive in appendix A the dependence of the downstream density and temperature $(n, T)$ on the initial density and the Lorentz factor, $\left(n_{0}, \gamma_{0}\right)$ in the non-relativistic regime, which is the relevant regime here. The relations derived in (A 7) enable to derive the conditions for the downstream plasma to be collisional. For quantum plasma, the boundary $n=6.3 \times 10^{22} \mathrm{~cm}^{-3}$ is equivalent to

$$
n_{0}=6.3 \times 10^{22}\left(\frac{\hat{\gamma}-1}{\hat{\gamma}+1}\right) \mathrm{cm}^{-3},
$$

where $\hat{\gamma}$ is the adiabatic index. If the temperature in the downstream is low, $T<T_{F}$, the plasma in the downstream region is degenerate for initial density $n_{0}$ larger than this value. 


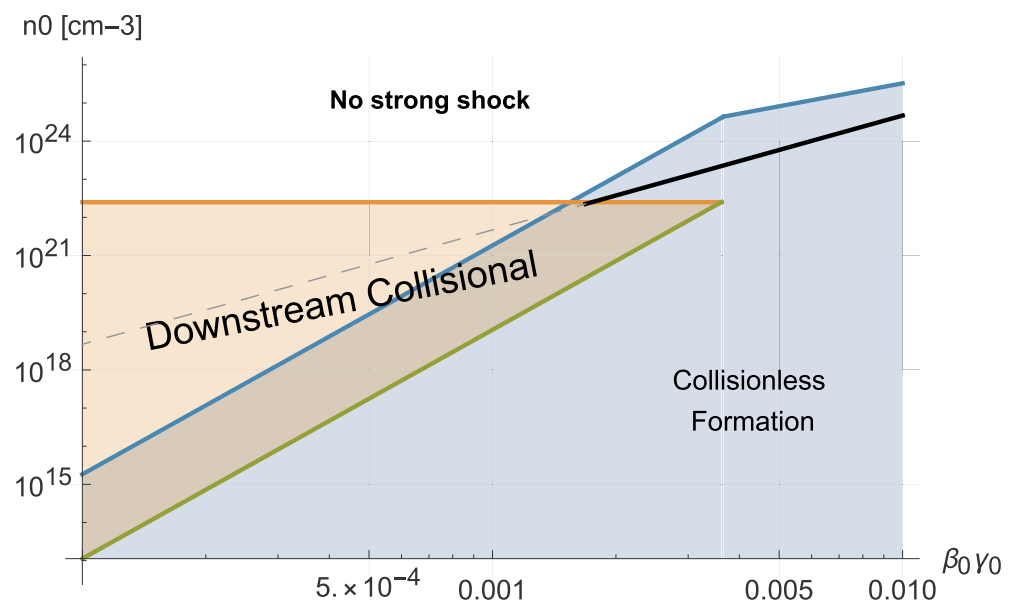

FIGURE 5. If the pair plasmas shells have their initial momenta and densities $\left(\beta_{0} \gamma_{0}, n_{0}\right)$ located inside the pale orange triangle, the downstream is collisional and the resulting shock cannot accelerate particles. In producing this plot, we consider $\hat{\gamma}=5 / 3$. The bold black line pictures the no strong shock condition at $T=0$ discussed in $\S 4$. At $T<T_{F}$, no strong shock forms above this line, because the speed of sound becomes larger than the collision speed.

For classical plasma, the boundary is determined by the condition $k_{B} T=q^{2} n^{1 / 3}$ (see (2.16)). Using (A 7), this relation can be written as $\left(\gamma_{0}, n_{0}\right)$

$$
\left.\begin{array}{l}
m_{e} v_{0}^{2}=q^{2}\left(n_{0} \frac{\hat{\gamma}+1}{\hat{\gamma}-1}\right)^{1 / 3} \\
\Rightarrow n_{0}=N^{*}\left(\frac{\hat{\gamma}-1}{\hat{\gamma}+1}\right) \beta_{0}^{6},
\end{array}\right\}
$$

where $N^{*}=4.5 \times 10^{37} \mathrm{~cm}^{-3}$ has been defined in (2.10). The conditions set in (2.17), (2.18) are equal at

$$
\beta_{0}=\left(\frac{8}{9 \pi^{4}}\right)^{1 / 6} \alpha=0.0033 \ll 1,
$$

justifying the non-relativistic treatment.

The boundaries defined by (2.17), (2.18) are shown in figure 5, together with the criterion for collisional or collisionless shock formation. If the initial shells are located inside the pale orange triangle, the downstream of the resulting shock is collisional (fluid); outside of this regime, it is collisionless.

It is noteworthy that there is a region of the phase space in which the shock formation is mediated by collisionless instabilities, while the downstream of the resulting shock is collisional. This region is enclosed between the blue and the green lines in figure 5. Comparing (2.15), (2.9) and (2.18), one sees that these two lines are almost exactly parallel, and are separated by a factor

$$
\frac{\text { Blue frontier }}{\text { Green frontier }} \sim \frac{128}{\pi} \frac{\hat{\gamma}+1}{\hat{\gamma}-1}=163 \text { for } \hat{\gamma}=5 / 3 \text {. }
$$


The upper bounds of the weakly coupled domain displayed on figure 4 translate to upper bounds in figure 5. If the colliding shells have initially either $\beta_{0}>0.0033\left(v_{0}>\right.$ $1003 \mathrm{~km} \mathrm{~s}^{-1}$ ) or $n_{0}>1.57 \times 10^{22} \mathrm{~cm}^{-3}$ (for $\hat{\gamma}=5 / 3$ ), then the downstream region of the resulting shock is always collisionless. In both cases, particle acceleration can occur.

\section{Electron/proton plasmas encounters}

We now adapt the previous results for the case of proton/electron plasmas. The overall dynamics of the system in this scenario is determined by the interaction of the protons. Following the structure of the preceding sections, we first assess the binary collision frequency before turning to the maximum growth rate.

\subsection{Inter-shell collision frequency}

The classical impact parameter for close binary Coulomb collisions in electron-proton plasma reads $b_{C}=q^{2} /\left(\gamma_{r} m_{p} v_{r}^{2}\right)$, where $m_{p}$ is the proton mass (see (2.1)). The quantum impact parameter in this scenario is $b_{Q}=\hbar / p=\hbar /\left(\gamma_{r} v_{r} m_{p}\right)$ (see (2.3)). The close collision frequency between protons of two different shells is therefore

$$
v_{s s}=n_{0} v_{r} \pi b^{2}=n_{0} \frac{2 \beta_{0}}{1+\beta_{0}^{2}} c \pi \max \left(b_{C}, b_{Q}\right)^{2} .
$$

One thus finds that in this scenario as well, the equality $b_{C}=b_{Q}$ is reached for $\beta_{r}=$ $\alpha \sim 1 / 137$.

\subsection{Growth rate for the collisionless interaction}

In the collisionless regime, the counter-streaming electrons first turn unstable as the shells start overlapping. Once the electronic instability has saturated, the counter-streaming protons turn unstable (Novo et al. 2015). In the relativistic regime, the most unstable mode of the counter-streaming protons over the bath of electrons is still the Weibel instability, with a maximum growth rate given by (Shaisultanov, Lyubarsky \& Eichler 2012)

$$
\delta=2 \frac{\beta_{0}}{\sqrt{\gamma_{0}}} \omega_{p p}
$$

This result is identical to that in (2.7), after replacing the electron plasma frequency $\omega_{p e}$ by the proton plasma frequency $\omega_{p p}^{2}=4 \pi n_{0} q^{2} / m_{p}$.

In the non-relativistic regime, the same pattern occurs. Electrons are stopped first before the counter-streaming protons become unstable over the bath of electrons. In the limit of small velocity, $\beta_{0} \ll 1$, the temperature of this electron bath approaches zero since its thermal energy originates from the initial kinetic energy of the electron beams. The fastest growing mode is found with a $\boldsymbol{k}$ aligned with the flow (Bret et al. $2010 b)$. The dispersion equation for the interaction is derived in appendix $\mathrm{B}$ and reads,

$$
\frac{2}{x^{2}}+\frac{R}{(Z-x)^{2}}+\frac{R}{(x+Z)^{2}}=1
$$




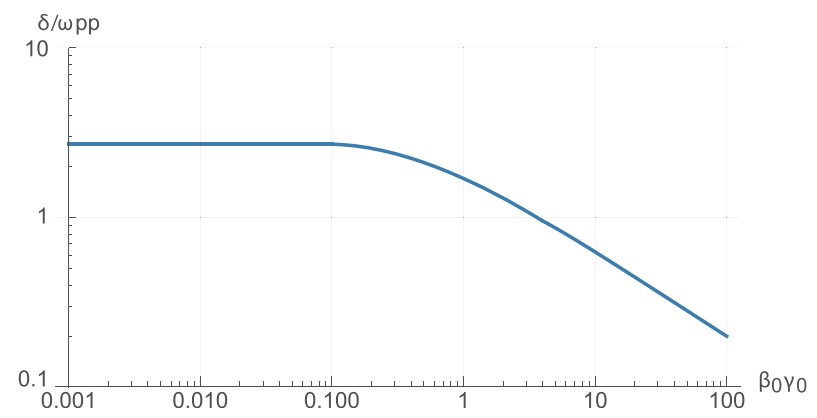

FIGURE 6. Growth rate of the fastest growing unstable mode in terms of $\beta_{0} \gamma_{0}$ for electron/proton plasmas interaction. In producing this plot, we took the mass ratio as $R=m_{e} / m_{p}=1 / 1836$. We interpolated between the non-relativistic (3.4) and the relativistic (3.2) regimes.

where $x=\omega / \omega_{p e}, Z=k v_{0} / \omega_{p e}$ and $R=m_{e} / m_{p}$ is the mass ratio (note that the frequency is measured in units of the electron plasma frequency). This equation is solved in appendix $\mathrm{B}$, yielding the maximum growth rate for this regime,

$$
\delta \sim \frac{\sqrt{3}}{2^{7 / 6}} R^{1 / 3} \omega_{p e}=\frac{\sqrt{3}}{2^{7 / 6}} R^{-1 / 6} \omega_{p p} .
$$

In both the relativistic (3.2) and non-relativistic (3.4) regimes, the growth rate $\delta$ is linear in $\omega_{p p}$, namely it admits the form $\delta=X \omega_{p p}$. In order to fill the gap between these regimes, we have implemented a simple first-order interpolation scheme. We point out that a more accurate fluid model attributing to the electron bath a temperature $3 k_{B} T \sim\left(\gamma_{0}-1\right) m c^{2}$ gives very similar results. Nevertheless, we consider the presentation of the fluid model unnecessary because of (i) its length, (ii) the small amount of additional precision it brings, (iii) the secondary relevance of this point with respect to the main theme of this work and (iv), the fact that the regime corresponding to this interpolation $\left(\beta_{0} \gamma_{0} \sim 1\right)$ pertains to densities larger than $10^{40} \mathrm{~cm}^{-3}$ (see figure 7).

The results of the growth rate are presented in figure 6. When comparing to the growth rate for a pair plasma in figure 2, we find that the local extremum has been lost. This feature comes from the main difference between the two settings, namely that for the electron/proton plasma, the proton Weibel instability grows over a bath of electrons.

\subsection{Comparing the collision rate $v_{s s}$ and the instability growth rate $\delta$}

We continue with the roadmap of the first part, comparing the time scales for collisional and collisionless interactions in the electron/proton case. In this scenario, we identify four separate regimes.

(i) For $\beta_{r}<\alpha=1 / 137$, equation (3.1) with $\max \left(b_{C}, b_{Q}\right)=b_{C}$ is compared with (3.4). One obtains

$$
\frac{v_{s s}}{\delta} \sim \frac{\sqrt{\pi / 3}}{42^{5 / 6}} \sqrt{\frac{n_{0}}{N^{*}}} R^{5 / 3} \frac{\left(1+\beta_{0}^{2}\right)^{3}}{\beta_{0}^{3}\left(2 \gamma_{0}^{2}-1\right)^{2}},
$$

where $N^{*}$ has been defined in (2.10). 


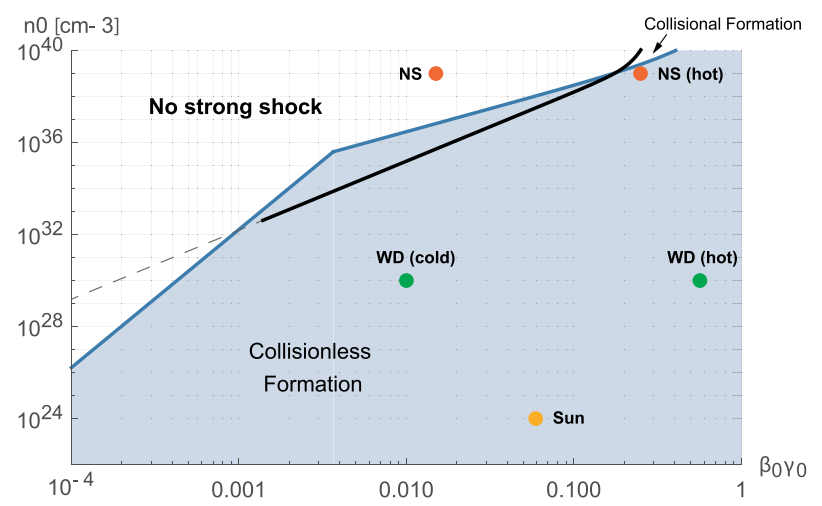

FIgURE 7. Frontier between the collisional and the collisionless domains for shock formation in the case of electron/proton plasmas. The coloured regions pertain to astrophysical scenarios discussed in $\S 5$. The bold black line pictures the no strong shock condition discussed in $\S 4$. At $T<T_{F}$, no strong shock forms above this line, as the speed of sound becomes larger than the collision speed.

(ii) For $\beta_{r}>\alpha=1 / 137$, yet $\beta_{0}$ still non-relativistic, equation (3.1) with $\max \left(b_{C}, b_{Q}\right)=$ $b_{Q}$, is to be compared with (3.4). One obtains,

$$
\frac{v_{s s}}{\delta} \sim 2^{-5 / 6} \sqrt{\frac{\pi}{3}} \sqrt{\frac{n_{0}}{N_{1}^{*}}} R^{5 / 3} \frac{\left(1+\beta_{0}^{2}\right)}{\beta_{0}\left(2 \gamma_{0}^{2}-1\right)^{2}}
$$

where $N_{1}^{*}$ has been defined in (2.12).

(iii) In the intermediate regime when $\beta_{0}$ approaches unity, the value of the growth rate has been interpolated (see figure 6), $\delta=X \omega_{p p}$. One chooses $\max \left(b_{C}, b_{Q}\right)=b_{Q}$ since $\beta_{r}>\alpha$ to obtain

$$
\frac{v_{s s}}{\delta} \sim \frac{\sqrt{\pi}}{X} \sqrt{\frac{n_{0}}{N_{1}^{*}}} R^{3 / 2} \frac{\beta_{0}}{\left(\beta_{0}^{2}+1\right) \beta_{r}^{2} \gamma_{r}^{2}} .
$$

(iv) In the relativistic regime, equation (3.1) with $\max \left(b_{C}, b_{Q}\right)=b_{Q}$, is to be compared with (3.2). One obtains,

$$
\frac{v_{s s}}{\delta} \sim \frac{\sqrt{\pi}}{16} \sqrt{\frac{n_{0}}{N_{1}^{*}}} R^{3 / 2} \frac{2\left(1+\beta_{0}^{2}\right) \sqrt{\gamma_{0}}}{\beta_{0}^{2}\left(2 \gamma_{0}^{2}-1\right)^{2}} .
$$

The frontier between the collisional and the collisionless domains for shock formation is displayed in figure 7 .

\subsection{Conditions on $n_{0}$ and $\gamma_{0}$ for a collisionless downstream}

The strongly coupled regime in the $(T, n)$ parameter space is similar to the one shown in figure 4 . Replacing the electron mass by the proton mass in the calculations yields a threshold density for weakly coupled degenerate plasmas of

$$
n>\frac{8}{9 \pi^{4}} \frac{m_{p}^{3} q^{6}}{\hbar^{6}}=3.88 \times 10^{32} \mathrm{~cm}^{-3} .
$$

Note that for classical plasmas, the weakly coupled regime still demands $k_{B} T>q^{2} n^{1 / 3}$. 


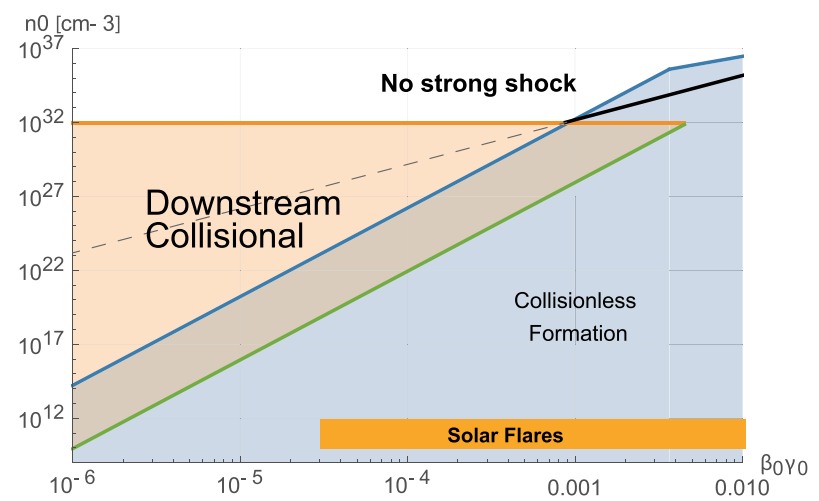

FIGURE 8. If the electron/proton plasma shells have their initial $\left(\beta_{0} \gamma_{0}, n_{0}\right)$ located inside the pale orange triangle, the downstream is collisional and the resulting shock cannot accelerate particles. We considered $\hat{\gamma}=5 / 3$. The coloured region pertains to the solar flares scenario discussed in $\S 5$. The bold black line pictures the no strong shock condition discussed in $\S 4$. At $T<T_{F}$, no strong shock forms above this line, as the speed of sound becomes larger than the collision speed.

The calculations conducted in appendix A to determine $n\left(n_{0}, \gamma_{0}\right)$ and $T\left(n_{0}, \gamma_{0}\right)$ are straightforwardly adapted. Equations (A 7) now read $k_{B} T=(1 / 2) m_{p} v_{0}^{2}$ and $n=(\hat{\gamma}+$ $1) /(\hat{\gamma}-1) n_{0}$, where the $1 / 2$ factor in the first equation accounts for the fact that the initial kinetic energy of the electrons is negligible compared to that of the protons $\left(k_{B} T \sim\left(m_{p} v_{0}^{2}\right) / 2\right)$.

The downstream of the formed shock will therefore be collisional if

$$
n_{0}<3.88 \times 10^{32} \frac{\hat{\gamma}-1}{\hat{\gamma}+1} \mathrm{~cm}^{-3}
$$

when degenerate, and

$$
n_{0}>\frac{1}{8}\left(\frac{\hat{\gamma}-1}{\hat{\gamma}+1}\right)\left(\frac{m_{p} c^{2}}{q^{2}}\right)^{3} \beta_{0}^{6}=3.47 \times 10^{46} \frac{\hat{\gamma}-1}{\hat{\gamma}+1} \beta_{0}^{6},
$$

when classical.

The corresponding region is pictured in figure 8. The intersection of the two strongly coupled limits is found at the same initial velocity as for the pair plasma, since the relevant value of $\beta_{0}(2.19)$ does not depend on the mass.

\section{About the small velocity spread regime: the 'no strong shock' condition}

In the calculations presented above, we neglected any velocity spread $\Delta v$ within the shells, that is, we assumed $\Delta v \ll v_{r}$. It is noteworthy that the speed of sound $c_{s}$ is proportional to $\Delta v$ (with a proportionality constant of order unity). Formally, the Riemann problem, consisting of two symmetric fluids colliding, always gives rise to two counter-propagating shock waves (see Landau \& Lifshitz (2013b) p. 362, or Zel'dovich \& Raizer (2002) p. 89). But as shown in appendix C by (C 8), for $\mathcal{M}_{0} \equiv v_{0} / c_{s} \ll 1$, the compression ratio of these shocks is $r \sim 1+\mathcal{M}_{0}$. And since the distribution of accelerated particles is a power law of index $q \propto(r-1)^{-1}$ (Blandford $\&$ Ostriker 1978), such weak shocks will not be efficient particle accelerators. 
Therefore, the equality $\Delta v \propto c_{s}=v_{r}$, which marks the limit of our calculations, also indicates the limit beyond which no strong shock forms, hence particle acceleration does not occur. Let us now further assess this limit.

Cold plasmas having $T<T_{F}$ are degenerate (see figure 4). In this regime, the speed of sound is a function of the Fermi temperature $T_{F}$, hence of the density. On the other hand, for $T>T_{F}$ the colliding shells are classical and the speed of sound becomes a function of the temperature. We shall now examine the limit $c_{s}=v_{r}$ first for degenerate plasma, and then in the classical regime.

\section{1. 'No strong shock' condition for cold, degenerate plasma}

Since the Fermi temperature increases with the density, for a given shell encounter speed $v_{r}$ the condition for strong shock formation, $v_{r}>c_{s}$ implies the existence of a critical (maximal) density, $n_{0, n s}{ }^{3}$ beyond which the encounter cannot produce a strong shock. At higher densities $n>n_{0, n s}$, the Fermi temperature $T_{F}$, hence the speed of sound, is larger than the relative speed of the collision.

In order to find the no strong shock condition, one needs to discriminate between the strongly coupled and the weakly coupled regimes. In the weakly coupled regime, simple analytical expressions exist in the non-relativistic (Newtonian) and the ultrarelativistic limits.

\subsubsection{Weakly coupled plasma regime}

The general expressions for the energy density and the pressure in a degenerate Fermi gas are given by (Landau \& Lifshitz 2013a),

$$
\begin{gathered}
u=\frac{c}{8 \pi^{2} \hbar^{3}}\left\{p_{F}\left(2 p_{F}^{2}+m^{2} c^{2}\right) \sqrt{p_{F}^{2}+m^{2} c^{2}}-(m c)^{4} \sinh ^{-1}\left(\frac{p_{F}}{m c}\right)\right\}, \\
P=\frac{c}{8 \pi^{2} \hbar^{3}}\left\{p_{F}\left(\frac{2}{3} p_{F}^{2}-m^{2} c^{2}\right) \sqrt{p_{F}^{2}+m^{2} c^{2}}+(m c)^{4} \sinh ^{-1}\left(\frac{p_{F}}{m c}\right)\right\},
\end{gathered}
$$

where

$$
p_{F}=\left(3 \pi^{2}\right)^{1 / 3} n^{1 / 3} \hbar
$$

is the Fermi momentum, and $m$ is the particle mass (electron or proton).

Simple expressions which enable exact analytical calculations exist in both the Newtonian and the ultra-relativistic limits. In the Newtonian limit, $p_{F} \ll m c$, equations (4.1), (4.2) are approximated by

$$
u(N . R .) \simeq \frac{3\left(3 \pi^{2}\right)^{2 / 3}}{10} \frac{\hbar^{2}}{m} n^{5 / 3}, \quad P(N . R .)=\frac{2}{3} u(N . R .) .
$$

On the other hand, in the ultra-relativistic limit, $p_{F} \gg m c$ one obtains (Chandrasekhar 1931),

$$
u(\text { rel. }) \simeq \frac{3}{8}\left(\frac{3}{\pi}\right)^{1 / 3} h c n^{4 / 3}, \quad P(\text { rel. })=\frac{u(\text { rel. })}{3} .
$$

The general expression for the speed of sound in a degenerate Fermi gas, which is correct in both the Newtonian and relativistic regimes is $c_{s}^{2}=c^{2}(\mathrm{~d} P / \mathrm{d} \bar{u})$, where $\bar{u}=$

${ }^{3}$ The subscript 'ns' in $n_{0, n s}$ stands for 'no shock'. 
$n m c^{2}+u$. In the Newtonian regime, $u \ll n m c^{2}$, this reduces to $c_{s}^{2}=p_{F}^{2} / 3 m^{2}$, while in the ultra-relativistic regime $c_{s}^{2}=c^{2} / 3$, similar to the well-known result obtained for classical gas.

In order to find the criterion for strong shock formation, it is easier to work in the comoving frame of one of the colliding shells. The comoving density $n$ is related to the laboratory-frame density $n_{0}$ by $n=n_{0} / \gamma_{0}$. In this frame the second shell is approaching at velocity $v_{r}$, given by (2.2). Equating this velocity with the speed of sound, $c_{s}$, one finds the maximal density that allows formation of strong shocks. In the Newtonian regime this density is

$$
n_{0, n s}=\frac{8 \sqrt{3}}{\pi^{2}}\left(\frac{m c}{\hbar}\right)^{3} \frac{\beta_{0}^{3}}{\left(1+\beta_{0}^{2}\right)^{3}} \gamma_{0} .
$$

In the relativistic regime, equating the colliding speed $v_{r}$ with the sound speed $c_{s}=$ $c / \sqrt{3}$ results in an asymptotic value of $\beta_{0}$,

$$
\beta_{0}=\sqrt{3}-\sqrt{2} \sim 0.32 \Rightarrow \beta_{0} \gamma_{0}=\frac{1}{2} \sqrt{\sqrt{6}-2} \sim 0.33 .
$$

We thus conclude that at faster shell velocities, strong shock waves will always form.

\subsubsection{Strongly coupled plasma regime}

At sufficiently low temperatures and low densities, namely $n_{0}<6.3 \times 10^{22} \mathrm{~cm}^{-3}$ (i.e. Fermi energy $E_{F}<q^{2} n^{1 / 3}$ ) the plasma particles are strongly coupled. In this regime, the Fermi energy is lower than the Coulomb potential. To the best of our knowledge, there is no simple expression for the speed of sound in this regime, which comprises warm dense matter (solid density at eV temperatures - see Fortney \& Nettelmann (2010) and references therein) and condensed matter. These limitations, however, do not substantially limit the breath of the present work, because shocks formed in such regimes are definitively collisional, hence clearly poor particle accelerators. We therefore represent the no strong shock boundary in this regime using dashed lines in figures $3,5,7$ and 8 .

The no strong shock criterion is plotted in thick black lines in figures 3 and 5 for the pair plasma scenario. Strong shocks cannot form above these lines. In the case of an electron/proton plasma, these calculations can be straightforwardly adapted replacing the electron mass by the proton mass. The corresponding criterion is plotted by thick black lines in figures 7 and 8 .

\section{2. 'No strong shock' condition in the classical plasma regime}

As long as $T<T_{F}(n)$, the shells can be regarded as cold, and the degenerate results at $\mathrm{T}=0$ apply. However, at sufficiently low densities, $T>T_{F}(n)$ and the speed of sound varies with the temperature rather than the density.

In this classical regime, the speed of sound is given by $c_{s}=c \sqrt{(\partial P / \partial u)_{s}}$, where $u$ is the energy density. Simple analytical expressions exist in the non-relativistic $\left(T \ll m c^{2}\right)$ and relativistic $\left(T \gg m c^{2}\right)$ limits:

$$
c_{s}= \begin{cases}\sqrt{\hat{\gamma} \frac{k_{B} T}{m}} & \left(T \ll m c^{2}\right), \\ \frac{c}{\sqrt{3}} & \left(T \gg m c^{2}\right),\end{cases}
$$

where $\hat{\gamma}$ is the adiabatic index. 


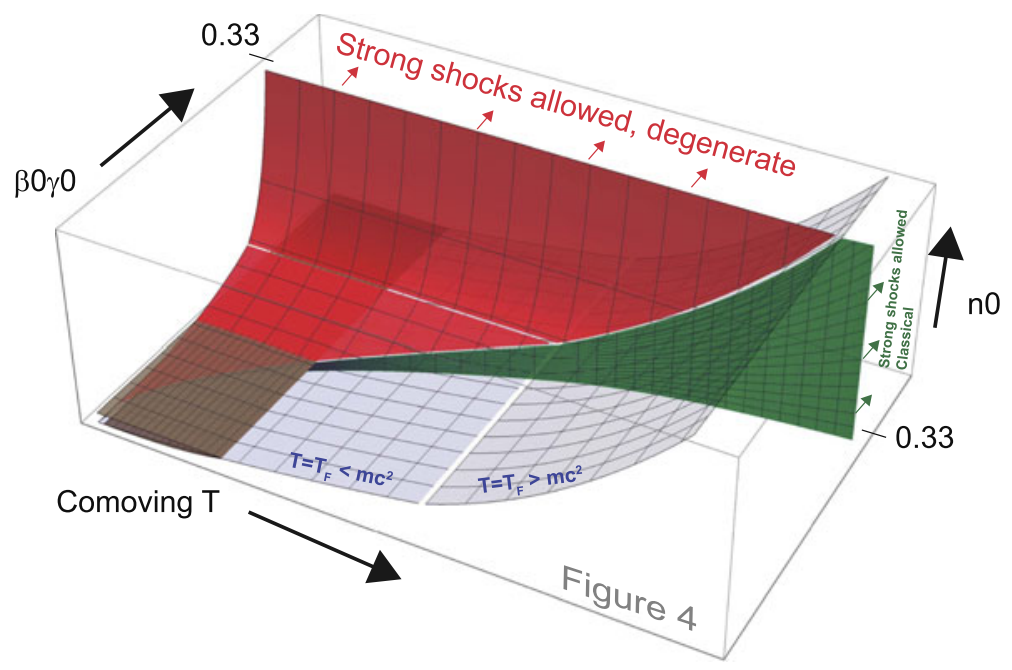

FIGURE 9. Sketch (not to scale) of the various domains involved in the article, in the phase space of parameters $\left(n_{0}, \beta_{0}, T\right)$. Strong shock formation is only allowed for systems located behind the red surface, or behind the green surface. Only weak shocks are formed in the rest of the parameter space, because the speed of sound exceeds the speed of the collision. Figure 4 corresponds to a two-dimensional cut at $\beta_{0} \gamma_{0}=0$, of this three-dimensional plot.

Similar to the degenerate case, in order for a strong shock to form, the requirement is $v_{r}>c_{s}$. Using (2.2), one finds that for low, non-relativistic temperatures, the minimum velocity scales as $\beta_{0} \propto \sqrt{T}$. For hot (relativistic) plasmas, the speed of sound is the same in both the classical and the degenerate regimes, and therefore the minimum value of $\beta_{0}$ that enables the production of strong shocks saturates to the value of $\beta_{0}$ given by (4.7). This coincidence of the degenerate and classical values of the minimum of $\beta_{0}$ allowed for strong shock formation was to be expected, since both merge for $T=T_{F}$.

\subsection{Complete parameter space region for strong shock formation}

The results of this section are summarized in figure 9. This figure generalizes the results presented in figures $3,5,7$ and 8 by adding a third dimension, namely, the comoving shell temperature.

The purple surface pictures systems with $T=T_{F}$. Above it, the plasma is degenerate, while below it is classical. As is evident, the slope breaks at $k_{B} T=m c^{2}$, as at lower temperatures the plasma is non-relativistic and $T_{F} \propto n^{2 / 3}$, while in the relativistic limit $T_{F} \propto n^{1 / 3} \rightarrow n \propto T_{F}^{3}$. This purple surface divides the parameter space into two regimes: above this surface, $T<T_{F}$ and the plasma is degenerate, while below this surface, $T>T_{F}$ and the plasma is classical.

The two brown surfaces represent the $k_{B} T=q^{2} n_{0}^{1 / 3}$ and $k_{B} T_{F}=q^{2} n_{0}^{1 / 3}$ limits. Systems located between these two surfaces are strongly coupled. These surfaces therefore represent a three-dimensional extension of the results presented in figure 4 (red lines).

The red/green surfaces picture the 'no strong shock' condition $\left(v_{r}<c_{s}\right)$ in degenerate/classical plasmas, respectively. In both regimes, as long as the plasma is in the Newtonian regime, increasing the temperature requires an increase in the 
collision velocity $\beta_{0}$ as $\beta_{0} \propto T^{1 / 2}$ in order to enable the formation of strong shocks. Thus, strong shocks can only be formed 'outside' of the pictured surfaces.

As the density increases, the Fermi temperature becomes relativistic, $T_{F}>m c^{2}$, and the condition on the collision velocity saturates at $\gamma_{0} \beta_{0} \simeq 0.33$ (see (4.7)). At higher collision speeds, strong shock could always form, regardless of the initial densities, the plasma temperatures or the nature of the plasma, being it either classical or degenerate.

\section{Astrophysical implications}

A key difference between collisional and collisionless shocks rely on the (theoretical) ability of the latter to both accelerate particles to high energies as well as generate strong magnetic fields. These phenomena cannot occur in collisional shocks, due to the rapid thermalization of the plasma in the downstream region, which suppresses the growth of any seed magnetic fields, as well as thermalizes energetic particles (e.g. Longair 2011).

Relativistic outflows in the form of astronomical jets are most easily observed at the exterior of many astronomical objects, ranging from supernova, X-ray emitting binaries (neutron stars or black holes) (Fender 2006, 2016), gamma-ray bursts (GRBs) (Mészáros 2006; Pe'er 2015) or supermassive black holes in active galactic nuclei (AGNs) (Begelman, Blandford \& Rees 1984; Urry \& Padovani 1995). While these objects inevitably involve shock waves, the low environmental densities imply that these shocks are formed collisionless, and so are their downstream regions.

The situation is somewhat more complicated in the interior of the various astronomical objects. We plot in figure 7 typical values of the interior density as well as the normalized velocities associated with random fluctuations of different objects. The velocities are derived based on the temperature estimate in the interior of the different objects, considering that forming a shock requires motions faster than the thermal velocity. We consider the solar interior, cooled white dwarfs (WD) (whose temperatures vary in the range $3 \times 10^{5}-10^{9} \mathrm{~K}$ ) (Shapiro \& Teukolsky 1983), and neutron stars (NS), having characteristic temperatures of $0.1-30 \mathrm{MeV}$ (Lattimer \& Prakash 2007). While shock waves that are generated in the interior of main sequence stars and WDs are always in the collisionless regime, shocks that can potentially form in the interior of NS can in principle be generated in the collisional regime, however they may fall into the 'no shock' parameter space region. As these shocks propagate outwards, they will eventually propagate towards the lower density and potentially higher velocity regime, and may therefore move into the collisionless regime of the parameter space.

Pair dominated plasmas are expected to occur in a few astronomical scenarios. One of the widely discussed scenarios is that of pair instability supernovae. Massive stars $\left(M \gtrsim 100 M_{\odot}\right)$ form large helium cores that reach carbon ignition with masses in excess of $\sim 45 M_{\odot}$. After helium burning, cores of this mass will encounter the electronpositron pair instability, collapse and ignite oxygen and silicon burning explosively. If explosive oxygen burning provides enough energy, the result is a "pair instability supernova' (Bond, Arnett \& Carr 1984; Heger \& Woosley 2002). In recent years, there were several observational evidence for this mechanism, e.g. in SN2006gy (Smith et al. 2007) or SN2007bi (Gal-Yam et al. 2009). In figure 3, we plot possible parameter space values for this scenario.

A second scenario is that of gamma-ray bursts (GRBs). The leading model to explain the observed variable light curves in these objects, the GRB 'fireball' model 
(Rees \& Meszaros 1994), invokes internal shocks. These shocks can in principle occur at radii as small as a few times the Schwarzschild radius of $\sim 10 M_{\odot}$ black hole, namely at $\gtrsim 10^{7} \mathrm{~cm}$. The densities are similar to those in the interior of massive stars. As these shocks occur below the photosphere, a significant number of pairs are created (Beloborodov 2017). Equilibration between pair production and annihilation results in density ratio of $n_{ \pm} / n_{e} \sim 10$ (Pe'er \& Waxman 2004). The typical values of density and velocity in this scenario is similar to that of pair instability supernovae, and is similarly plotted in figure 3 .

We thus conclude that in these objects, the shock waves can initially be formed as collisional, although as they propagate outward, they become collisionless. As a consequence, the time available for particle acceleration and magnetic field generation in these shock waves could be limited.

Another environment of interest is that of solar flares. With typical electron densities of $\sim 10^{10}-10^{12} \mathrm{~cm}^{-3}$ (Aschwanden 2002) and typical velocities in the range 30-10 $\mathrm{km} \mathrm{s}^{-1}$ (Aschwanden 2002; Jeffrey, Fletcher \& Labrosse 2017), the resulting shock waves are expected to be formed collisionless, but the downstream region of the low velocity shocks (at least, initially) could potentially be collisional. While the flares will eventually propagate into a lower density region, these conditions limit their ability to accelerate particles at their initial phases. The corresponding parameters space values are plotted on figure 8.

\section{Conclusions}

This article deals with the properties of the shock waves that are formed when two symmetrical plasmas run into each other, and in particular with the question of collisionality. We assessed the answers to two questions: (i) When is the interaction mediated by close Coulomb binary collisions or collective plasma instabilities? (ii) Once a shock has been formed, when is its downstream collisionless?

The answer to the first question is given in figure 3 for pair plasmas and figure 7 for electron-proton plasmas. The answer to the second question is presented in figures 5 and 8 for pair and electron-proton plasmas respectively.

As we showed here, the switch from the collisional to collisionless regime bears consequences for the time scale of the shock formation. In the collisionless regime, the shock formation time is determined by the growth rate of the unstable interaction between the two shells. In the collisional regime, on the other hand, the shock formation time is set by the frequency of close binary collisions.

Moreover, a shock for which the formation not mediated by collisionless plasma instabilities will not inherit the downstream electromagnetic patterns formed by these instabilities. Collisions of shells with curved boundaries may trigger downstream vorticity that could generate magnetic fields (Bond et al. 2017). But colliding planar shells like those considered here, will yield a field free downstream if the formation is collisional.

Understanding the properties of the downstream region (the second question outlined above) has the important consequence of determining the ability of the downstream region to accelerate particles. As discussed above, a collisional downstream leads to a suppression of particle acceleration. For two plasma shells initially located in the orange triangle plotted in figure 5 for pair plasmas, or figure 8 for electron/proton plasmas, the resulting shock will not be able to accelerate particles. As a result, the radiative signature of the system would be dramatically different than that of a system in which the downstream is collisionless. 
In $\S 5$, we discussed several astrophysical settings where a shock may be formed as collisional, and, during its propagation inside the object, its properties will be modified from a collisional to a collisionless medium. The key consequences of this transition is the limited ability of these shocks to accelerate particles to high energies and to generate magnetic fields. This implies stringent constraints on the abilities of these objects to be the sources of high energy cosmic rays, as well as modification of the resulting spectra. We leave a detailed study of the spectra expected under various conditions to future work. Similar situations can be found in the context of inertial confinement fusion (Zhang et al. 2017). While the medium considered here are spatially homogeneous, it would be worth studying how a shock transiting from one kind of medium to another, evolves.

In this work, we focused on 'classical' shocks, namely shocks that are mediated either by collective plasma effects or by Coulomb collisions. If shock waves occur in regions of high optical depth in which the mean free path is smaller than the shock size, they may be mediated by photons scattered back and forth in the upstream and downstream regions (Budnik et al. 2010; Beloborodov 2017; Lundman, Beloborodov \& Vurm 2018), provided that the radiation energy density dominates the energy density of the gas. This scenario is further expected to modify the shock properties; in particular, no particle acceleration is expected. We leave a full treatment of the properties of these radiative-mediated shocks to a future work. We do point out, however, that in all astrophysical implications considered in this work, namely neutron stars, white dwarfs and stellar interiors, radiative pressure is sub-dominant over the gas pressure, and thus the formed shock waves are not expected to be radiatively mediated. Radiatively mediated shocks are expected in stellar envelopes, stellar winds, supernovæ explosions, and possibly in gamma-ray bursts.

The encounter of partially ionized shells could be worthy of investigation. In such a setting, the outcome may sharply depend on the intra-shell coupling between the ionized and the neutral particles. If intra-shell collisions are rare, both components may act separately. The ionized part should form a collisionless shock on a time scale given by plasma instabilities. Meanwhile, the neutral component could form a shock on a time scale defined by the collision frequency between neutrals of different shells. But if both components are coupled, then the first one to form a shock may drag the other into the formation of a single, common, shock. Such scenarios will be studied in future works.

Future works could also focus on studying temperature effects. The present calculations are valid as long as the shells' velocity spread $\Delta v$ fulfils $\Delta v \ll v_{r}$. Larger spreads should affect the binary collisions frequencies, the maximum growth rates and also the Rankine-Hugoniot (RH) conditions used to determine the collisionality of the downstream. While such effects on the binary collision frequencies, or on the $\mathrm{RH}$ conditions are accessible, there are so far no analytical formulas available for the maximum growth rate in terms of the temperature and $\gamma_{0}$ (Bret, Gremillet \& Bénisti $2010 a$ ). Such progress is therefore a prerequisite before one can elaborate on larger temperature effects. Note however that as discussed in $\$ 4$, no strong shock should form when $\Delta v>v_{r}$, since the speed of sound becomes larger than the collision speed in this limit. Therefore, as far as the velocity spread is concerned, this work investigates the case $\Delta v / v_{r} \ll 1$, and the regime left to explore is simply $\Delta v / v_{r}$ of order unity.

\section{Acknowledgements}

A.B. acknowledges support by grant ENE2016-75703-R from the Spanish Ministerio de Educación. A.P. acknowledges support by the European Union Seventh Framework 
Program (FP7/2007-2013) under grant agreement no. 618499, and support from NASA under grant no. NNX12AO83G. A.B. thanks Gustavo Wouchuk, Roberto Piriz, Rony Keppens, Rolf Walder and Lorenzo Sironi for fruitful discussions.

\section{Appendix A. Determination of $n\left(n_{0}, \gamma_{0}\right)$ and $T\left(n_{0}, \gamma_{0}\right)$}

The fact that the collisional regime lies deep within the non-relativistic domain as is shown in figure 4, suggests that a non-relativistic treatment is appropriate. Once the shock is formed, the shock frame is well defined. In this frame, we use the subscripts ' 1 ' ('2') to describe upstream (downstream) quantities. In the non-relativistic regime and for zero upstream pressure $\left(P_{1}=0\right)$, the Rankine-Hugoniot $(\mathrm{RH})$ relations read (Zel'dovich \& Raizer 2002),

$$
\left.\begin{array}{c}
\frac{n_{2}}{n_{1}}=\frac{\rho_{2}}{\rho_{1}}=\frac{\hat{\gamma}+1}{\hat{\gamma}-1}, \\
\frac{v_{2}}{v_{1}}=\frac{\hat{\gamma}-1}{\hat{\gamma}+1} .
\end{array}\right\}
$$

Here, $\hat{\gamma}$ is the adiabatic index of the gas, $\rho_{1}, \rho_{2}, v_{1}, v_{2}$ are the mass densities, upstream and downstream velocities respectively. The $\mathrm{RH}$ relation for the downstream pressure $P_{2}$ leads to

$$
\begin{aligned}
P_{2} & =\rho_{1} v_{1}^{2}-\rho_{2} v_{2}^{2} \\
& =\rho_{1} v_{1}^{2}-\rho_{1} \frac{\hat{\gamma}+1}{\hat{\gamma}-1}\left(v_{1} \frac{\hat{\gamma}-1}{\hat{\gamma}+1}\right)^{2} \\
& =\rho_{1} v_{1}^{2} \frac{2}{\hat{\gamma}+1} .
\end{aligned}
$$

Since the plasma is non-relativistic, the upstream velocity as written in the downstream frame is simply $\beta_{0}=v_{0} / c$. We thus have,

$$
v_{0}=v_{1}-v_{2}=v_{1}-\frac{\hat{\gamma}-1}{\hat{\gamma}+1} v_{1}=\frac{2}{\hat{\gamma}+1} v_{1} \text {. }
$$

Using this result in (A 2) then gives,

$$
\begin{aligned}
P_{2} & =\rho_{1} v_{1}^{2} \frac{2}{\hat{\gamma}+1}=\rho_{1}\left(\frac{\hat{\gamma}+1}{2} v_{0}\right)^{2} \frac{2}{\hat{\gamma}+1} \\
& =\rho_{0} \frac{\hat{\gamma}+1}{2} v_{0}^{2}=2 n_{0} m_{e} \frac{\hat{\gamma}+1}{2} v_{0}^{2},
\end{aligned}
$$

where $\rho_{1}$ has been replaced by $\rho_{0}$ in the last line, as no relativistic boosting exists, while the factor 2 comes because there are $n_{0}$ electrons and $n_{0}$ positrons per unit volume.

Denoting the energy density by $u$, the downstream pressure $P_{2}$ reads,

$$
P_{2}=(\hat{\gamma}-1) \rho u=(\hat{\gamma}-1) n k_{B} T .
$$

One finally obtains,

$$
n k_{B} T=m_{e} \frac{\hat{\gamma}+1}{\hat{\gamma}-1} n_{0} v_{0}^{2}
$$



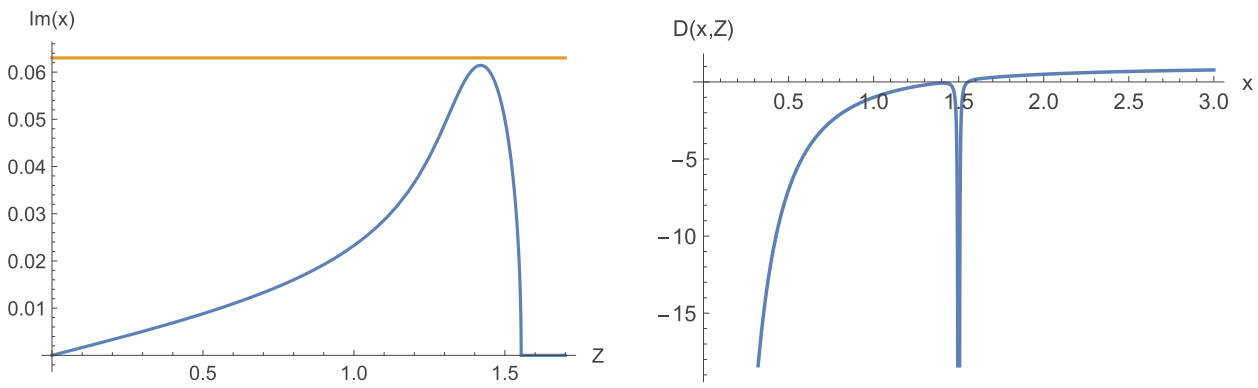

FIgURE 10. (a) Growth rate given by $\operatorname{Im}(x)$ with $x / D(x, Z)=0$ for $R=1 / 1836$. The orange line shows the result given by (3.4). (b) Plot of the dispersion function $D(x, Z)$ defined in (B 2), for $R=1 / 1836$ and $Z=1.5$.

namely

$$
\left.\begin{array}{l}
k_{B} T=m_{e} v_{0}^{2}, \\
n=n_{0} \frac{\hat{\gamma}+1}{\hat{\gamma}-1},
\end{array}\right\}
$$

which are the relations we need. The first one reads $k_{B} T=\left(2 m_{e} v_{0}^{2}\right) / 2$, which simply states that the downstream thermal energy originates from the upstream kinetic energy.

\section{Appendix B. Derivation of the dispersion (3.3)}

Suppose we have $a \in \mathbb{N}$ cold beams made of species of densities $n_{j}$, masses $m_{j}$ and velocities $\boldsymbol{v}_{j}=v_{j} \boldsymbol{e}_{z}$, the system being overall charge and current neutral. The dispersion equation for longitudinal waves with $\boldsymbol{k} \| \boldsymbol{e}_{z}$ reads (see Ichimaru (1973), p. 137),

$$
\sum_{j=1}^{a} \frac{\omega_{p j}^{2}}{\left(\omega-\boldsymbol{k} \cdot \boldsymbol{v}_{j}\right)^{2}}=1
$$

where $\omega_{p j}^{2}=4 \pi n_{j} q^{2} / m_{j}$ is the plasma frequency of specie $j$. The unstable system considered in $\$ 3.2$ accounts for 3 species: the 2 counter-streaming proton beams, and the cold electronic background. With the dimensionless variables used, (B 1) then gives,

$$
D(x, Z) \equiv \frac{2}{x^{2}}+\frac{R}{(Z-x)^{2}}+\frac{R}{(x+Z)^{2}}-1=0,
$$

which is (3.3).

This dispersion equation can be derived either using a multiple cold fluid model (Califano et al. 1998), or taking the kinetic dispersion equation for such waves, and considering a distribution function which is the sum of Dirac delta functions (as in Ichimaru (1973)).

The growth rate $\operatorname{Im}(x)$, with $x / D(x, Z)=0$, is plotted in figure $10(a)$ for $R=1 / 1836$. It reaches a maximum near $Z=\sqrt{2}$. This can be understood by noting that, since $R \ll 1$, the roots of the equation have to be close to the roots of the same equation, but with $R=0$, that is $x= \pm \sqrt{2}$. It follows that for $0<R \ll 1$, the numerators of the 

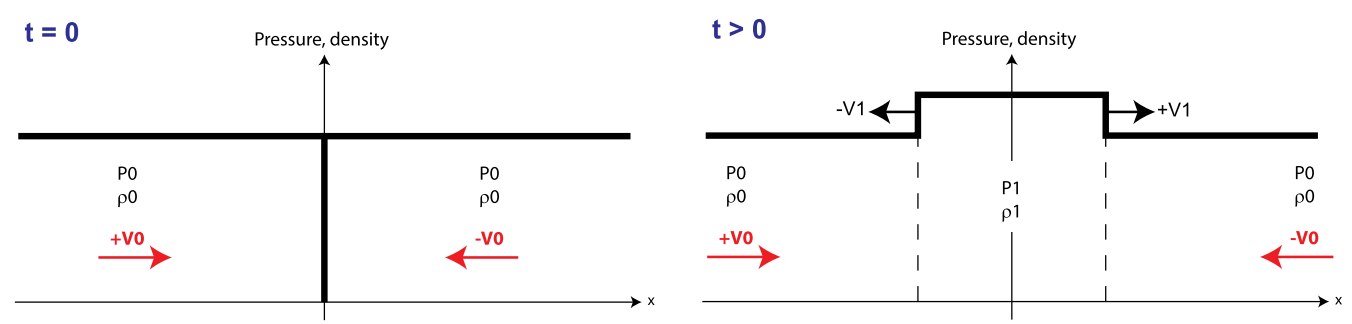

FIGURE 11. Riemann problem considered.

terms $\propto R$ have to be small if these terms are to bring a significant contribution to the equation. This implies in turn $Z \sim \pm \sqrt{2}$.

The dispersion function $D(x, Z)$ is plotted on figure $10(b)$ for $x>0, R=1 / 1836$ and $Z=1.5$. It is an even function of $x$, which is the reason why only the $x>0$ part is plotted. Let us now focus on the root located near $x=\sqrt{2}$. It is primarily determined by the terms $2 / x^{2}$ and $R /(x-Z)^{2}$ of the dispersion equation. We can therefore study the solution located near $x=\sqrt{2}$ by neglecting $R /(x+Z)^{2}$, that is, solving,

$$
\frac{2}{x^{2}}+\frac{R}{(Z-x)^{2}}=1
$$

We can solve approximately this equation following a method derived long ago (Bludman, Watson \& Rosenbluth 1960). Knowing that the maximum growth rate $\delta$ is found for $x \sim \sqrt{2}$, we write $x=\sqrt{2}+\mathrm{i} \delta$, with $\delta \in \mathbb{R}$. We then assume $|Z-\sqrt{2}| \ll|\delta|$ (which is later verified) so that (B 3 ) becomes,

$$
\frac{2}{(\sqrt{2}+\mathrm{i} \delta)^{2}}-\frac{R}{\delta^{2}}=1 \text {. }
$$

A Taylor expansion of the first term, and some straightforward algebra, gives the growth rate (3.4).

\section{Appendix C. Density jump of shocks formed a low collision velocity}

The Riemann problem pictured in figure 11 consists of two symmetric fluids colliding. It gives rise to 2 counter-propagating shock waves (see Landau \& Lifshitz (2013b) p. 362, or Zel'dovich \& Raizer (2002) p. 89). Because the efficiency of particle acceleration depends on the compression ratio $r$, let us compute $\rho_{1}$ in terms of $v_{0}, P_{0}, \rho_{0}$.

In the reference frame of the shock front, the upstream comes at $V_{0}+V_{1}$, and the downstream goes at $V_{1}$. We thus have,

$$
\begin{gathered}
\rho_{1} V_{1}=\rho_{0}\left(V_{1}+V_{0}\right), \\
\rho_{1}=\rho_{0} \frac{(\hat{\gamma}+1)\left(\mathcal{M}_{0}+M_{1}\right)^{2}}{(\hat{\gamma}-1)\left(\mathcal{M}_{0}+\mathcal{M}_{1}\right)^{2}+2},
\end{gathered}
$$

where the first equation is the conservation of matter, the second, the RankineHugoniot jump condition for the density, and

$$
\mathcal{M}_{0}=\frac{V_{0}}{\sqrt{\hat{\gamma} P_{0} / \rho_{0}}}
$$




$$
\mathcal{M}_{1}=\frac{V_{1}}{\sqrt{\hat{\gamma} P_{0} / \rho_{0}}} .
$$

Setting $r=\rho_{1} / \rho_{0}$, these equations read,

$$
\begin{gathered}
r=\frac{\mathcal{M}_{0}}{\mathcal{M}_{1}}+1, \\
r=\frac{(\hat{\gamma}+1)\left(\mathcal{M}_{0}+\mathcal{M}_{1}\right)^{2}}{(\hat{\gamma}-1)\left(\mathcal{M}_{0}+\mathcal{M}_{1}\right)^{2}+2} .
\end{gathered}
$$

These expressions must be solved for $r$ and $\mathcal{M}_{1}$, under the constraint $\mathcal{M}_{0}+\mathcal{M}_{1}>1$.

Eliminating $r$ gives a third-order polynomial which can be factored by $\left(\mathcal{M}_{0}+\mathcal{M}_{1}\right)$. The remaining second-order polynomial can be solved exactly. Its positive root is,

$$
\mathcal{M}_{1}=\frac{1}{4}\left[(\hat{\gamma}-3) \mathcal{M}_{0}+\sqrt{(\hat{\gamma}+1)^{2} \mathcal{M}_{0}^{2}+16}\right] .
$$

We have $\mathcal{M}_{1}\left(\mathcal{M}_{0}=0\right)=1$. In addition, it is easy to prove that $\forall \mathcal{M}_{0}, \partial\left(\mathcal{M}_{0}+\right.$ $\left.\mathcal{M}_{1}\right) / \partial \mathcal{M}_{0}>0$, so that $\mathcal{M}_{0}+\mathcal{M}_{1}>1$ is always verified.

Then $r=\rho_{1} / \rho_{0}$ follows from $r=1+\mathcal{M}_{0} / \mathcal{M}_{1}$.

For small impact velocities, namely $\mathcal{M}_{0} \ll 1$, we find

$$
\left.\begin{array}{c}
r=1+\mathcal{M}_{0}+O\left(\mathcal{M}_{0}^{2}\right), \\
\mathcal{M}_{1}=1+\frac{\hat{\gamma}-3}{4} \mathcal{M}_{0}+O\left(\mathcal{M}_{0}^{2}\right), \\
\mathcal{M}_{0}+\mathcal{M}_{1}=1+\frac{\hat{\gamma}+1}{4} \mathcal{M}_{0}+O\left(\mathcal{M}_{0}^{2}\right) .
\end{array}\right\}
$$

In the opposite limit $\mathcal{M}_{0} \gg 1$,

$$
\begin{aligned}
& r \rightarrow \frac{\hat{\gamma}+1}{\hat{\gamma}-1} \quad \text { (strong shock limit), } \\
& \left.\mathcal{M}_{1} \rightarrow \frac{\hat{\gamma}-1}{2} \mathcal{M}_{0}, \quad\right\} \\
& \mathcal{M}_{0}+\mathcal{M}_{1} \rightarrow \frac{\hat{\gamma}+1}{2} \mathcal{M}_{0} .
\end{aligned}
$$

\section{REFERENCES}

Aschwanden, M. J. 2002 Particle acceleration and kinematics in solar flares - A synthesis of recent observations and theoretical concepts (invited review). Space Science Rev. 101, 1-227. Ashcroft, N. W. \& Mermin, N. D. 1976 Solid State Physics. Holt, Rinehart and Winston.

Bale, S. D., Mozer, F. S. \& Horbury, T. S. 2003 Density-transition scale at quasiperpendicular collisionless shocks. Phys. Rev. Lett. 91, 265004.

Bamba, A., Yamazaki, R., Ueno, M. \& Koyama, K. 2003 Small-scale structure of the SN 1006 shock with chandra observations. Nature 589, 827-837.

Begelman, M. C., Blandford, R. D. \& Rees, M. J. 1984 Theory of extragalactic radio sources. Rev. Mod. Phys. 56, 255-351.

Bell, A. R. 1978 The acceleration of cosmic rays in shock fronts. I. Mon. Not. R. Astron. Soc. 182, 147-156. 
Beloborodov, A. M. 2017 Sub-photospheric shocks in relativistic explosions. Nature 838, 125.

BLANDFORD, R. \& EICHLER, D. 1987 Particle acceleration at astrophysical shocks: A theory of cosmic ray origin. Phys. Rep. 154, 1.

Blandford, R. D. \& OStRIKer, J. P. 1978 Particle acceleration by astrophysical shocks. Astrophys. J. 221, L29.

Bludman, S. A., Watson, K. M. \& Rosenbluth, M. N. 1960 Statistical mechanics of relativistic streams. ii. Phys. Fluids 3, 747.

Bond, D., Wheatley, V., Samtaney, R. \& Pullin, D. I. 2017 Richtmyer-meshkov instability of a thermal interface in a two-fluid plasma. J. Fluid Mech. 833, 332-363.

Bond, J. R., ARnetT, W. D. \& CARR, B. J. 1984 The evolution and fate of very massive objects. Nature 280, 825-847.

Bret, A., Gremillet, L. \& BÉnisti, D. $2010 a$ Exact relativistic kinetic theory of the full unstable spectrum of an electron-beam plasma system with maxwell-jüttner distribution functions. Phys. Rev. E 81, 036402.

Bret, A., Gremillet, L. \& Dieckmann, M. E. $2010 b$ Multidimensional electron beam-plasma instabilities in the relativistic regime. Phys. Plasmas 17, 120501.

Bret, A., Stockem, A., Fiúza, F., Ruyer, C., Gremillet, L., Narayan, R. \& Silva, L. O. 2013 Collisionless shock formation, spontaneous electromagnetic fluctuations, and streaming instabilities. Phys. Plasmas 20, 042102.

Bret, A., Stockem, A., Narayan, R. \& Silva, L. O. 2014 Collisionless weibel shocks: Full formation mechanism and timing. Phys. Plasmas 21 (7), 072301.

Budnik, R., Katz, B., Sagiv, A. \& WaXman, E. 2010 Relativistic radiation mediated shocks. Nature 725, 63-90.

Califano, F., Prandi, R., Pegoraro, F. \& Bulanov, S. V. 1998 Nonlinear filamentation instability driven by an inhomogeneous current in a collisionless plasma. Phys. Rev. E 58, 7837.

Chandrasekhar, S. 1931 The maximum mass of ideal white dwarfs. Astrophys. J. 74, 81.

Fender, R. 2006 Jets from X-ray binaries. In Compact Stellar X-ray Sources (ed. W. Lewin \& M. Van der Klis), Cambridge Astrophysics, pp. 381-420. Cambridge University Press.

FENDER, R. 2016 Disc-jet-wind coupling in black hole binaries, and other stories. Astron. Nachr. 337, 381.

Fortney, J. J. \& NetTElmanN, N. 2010 The interior structure, composition, and evolution of giant planets. Space Science Rev. 152, 423-447.

Gal-Yam, A., Mazzali, P., Ofek, E. O., Nugent, P. E., Kulkarni, S. R., Kasliwal, M. M., Quimby, R. M., Filippenko, A. V., Cenko, S. B., Chornock, R. et al. 2009 Supernova 2007bi as a pair-instability explosion. Astrophys. J. 462, 624-627.

Heger, A. \& Woosley, S. E. 2002 The nucleosynthetic signature of population III. Nature 567, 532-543.

Huntington, C. M., Fiuza, F., Ross, J. S., Zylstra, A. B., Drake, R. P., Froula, D. H., Gregori, G., Kugland, N. L., Kuranz, C. C., Levy, M. C. et al. 2015 Observation of magnetic field generation via the Weibel instability in interpenetrating plasma flows. Nat. Phys. 11, 173-176.

IChimaru, S. 1973 Basic Principles of Plasma Physics. W. A. Benjamin, Inc.

ICHIMARU, S. 1982 Strongly coupled plasmas: high-density classical plasmas and degenerate electron liquids. Rev. Mod. Phys. 54, 1017-1059.

JACKSON, J. D. 1998 Classical Electrodynamics. Wiley.

Jeffrey, N. L. S., Fletcher, L. \& Labrosse, N. 2017 Non-Gaussian velocity distributions in solar flares from extreme ultraviolet lines: A possible diagnostic of ion acceleration. Nature 836, 35 .

Landau, L. D. \& Lifshitz, E. M. 2013 a Course of Theoretical Physics, Statistical Physics. Elsevier.

LANDAU, L. D. \& Lifshitz, E. M. 2013b Fluid Mechanics. Elsevier.

LAtTimer, J. M. \& Prakash, M. 2007 Neutron star observations: Prognosis for equation of state constraints. Phys. Rep. 442, 109-165.

Longair, M. S. 2011 High Energy Astrophysics. Cambridge University Press. 
Lundman, C., Beloborodov, A. \& Vurm, I. 2018 Radiation-mediated shocks in gamma-ray bursts: Pair creation. Astrophys. J. 858, 7.

Marcowith, A., Bret, A., Bykov, A., Dieckmann, M. E., Drury, L. O. C., Lembège, B., Lemoine, M., Morlino, G., Murphy, G., Pelletier, G. et al. 2016 The microphysics of collisionless shock waves. Rep. Prog. Phys. 79, 046901.

Medvedev, M. V., Fiore, M., Fonseca, R. A., Silva, L. O. \& Mori, W. B. 2005 Long-time evolution of magnetic fields in relativistic gamma-ray burst shocks. Astrophys. J. 618, L75.

Mészáros, P. 2006 Gamma-ray bursts. Rep. Prog. Phys. 69, 2259-2321.

MilosaVlJeVIC, M. \& NAKAR, E. 2006 Weibel filament decay and thermalization in collisionless shocks and gamma-ray burst afterglows. Astrophys. J. 641, 978-983.

Novo, A. S., Bret, A., Fonseca, R. A. \& Silva, L. O. 2015 Shock formation in electron-ion plasmas: Mechanism and timing. Astrophys. J. Lett. 803 (2), L29.

PE'ER, A. 2015 Physics of gamma-ray bursts prompt emission. Adv. Astron. 2015, 907321.

PE'ER, A. \& WAXMAN, E. 2004 Prompt gamma-ray burst spectra: Detailed calculations and the effect of pair production. Nature 613, 448-459.

PetscheK, H. E. 1958 Aerodynamic dissipation. Rev. Mod. Phys. 30, 966-974.

ReEs, M. J. \& Meszaros, P. 1994 Unsteady outflow models for cosmological gamma-ray bursts. Astrophys. J. Lett. 430, L93-L96.

Rybicki, G. B. \& Lightman, A. P. 1979 Radiative Processes in Astrophysics. Wiley.

SAGDEEV, R. Z. 1966 Cooperative phenomena and shock waves in collisionless plasmas. Rev. Plasma Phys. 4, 23.

Sagdeev, R. Z. \& Kennel, C. F. 1991 Collisionless shock waves. Sci. Am. 264 (4), 106-115.

Schwartz, S. J., Henley, E., Mitchell, J. \& Krasnoselskikh, V. 2011 Electron temperature gradient scale at collisionless shocks. Phys. Rev. Lett. 107, 215002.

Shaisultanov, R., LyUbarsky, Y. \& Eichler, D. 2012 Stream instabilities in relativistically hot plasma. Astrophys. J. 744, 182.

Shapiro, S. L. \& Teukolsky, S. A. 1983 Black Holes, White Dwarfs and Neutron Stars: The Physics of Compact Objects, A Wiley-interscience Publication. Wiley.

Silva, L. O., Fonseca, R. A., Tonge, J. W., Dawson, J. M., Mori, W. B. \& Medvedev, M. V. 2003 Interpenetrating plasma shells: Near-equipartition magnetic field generation and nonthermal particle acceleration. Astrophys. J. Lett. 596, L121-L124.

Sironi, L. \& SPITKOVSKY, A. 2011 Particle acceleration in relativistic magnetized collisionless electron-ion shocks. Nature 726, 75-+.

Smith, N., Li, W., Foley, R. J., Wheeler, J. C., Pooley, D., Chornock, R., Filippenko, A. V., Silverman, J. M., QuimbY, R., Bloom, J. S. et al. 2007 SN 2006gy: Discovery of the most luminous supernova ever recorded, powered by the death of an extremely massive star like $\eta$ carinae. Nature 666, 1116-1128.

SPITKOVSKY, A. 2008 Particle acceleration in relativistic collisionless shocks: Fermi process at last? Astrophys. J. Lett. 682, L5-L8.

Urry, C. M. \& Padovani, P. 1995 Unified schemes for radio-loud active galactic nuclei. Publ. Astr. Soc. Pacific 107, 803-845.

Watson, K. M., Bludman, S. A. \& Rosenbluth, M. N. 1960 Statistical mechanics of relativistic streams. i. Phys. Fluids 3, 741.

Zel'dovich, Y. B. \& Raizer, Y. P. 2002 Physics of Shock Waves and High-temperature Hydrodynamic Phenomena. Dover Publications.

Zhang, W.-S., CAI, H.-B., Shan, L.-Q., Zhang, H.-S., GU, Y.-Q. \& ZHU, S.-P. 2017 Anomalous neutron yield in indirect-drive inertial-confinement-fusion due to the formation of collisionless shocks in the corona. Nucl. Fusion 57 (6), 066012. 